\title{
Erbium Doped Fiber Laser Mode Locked by Graphene in Carboxymethylcellulose Polymer Composite
}

\author{
Chengbo Mou ${ }^{1}$, Raz Arif ${ }^{1}$, Anatoly S. Lobach ${ }^{2}$, Nataliya G. Spitsina ${ }^{2}$, Valery A. Kazakov ${ }^{3}$, Sergei Turitsyn ${ }^{1}$, \\ Aleksey Rozhin ${ }^{1}$ \\ ${ }^{1}$ Aston Institute of Photonic Technologies, Aston University, Aston Triangle, Birmingham, United Kingdom, B4 7ET \\ ${ }^{2}$ Institute of Problems of Chemical Physics RAS, Ac. Semenov Av. 1, Chernogolovka, Moscow Region 142432, Russia \\ ${ }^{3}$ Federal State Unitary Enterprise, Keldysh Research Center, Onezhskaya 8, Moscow 125438, Russia \\ Corresponding email:mouc1@aston.ac.uk
}

\begin{abstract}
We have presented and demonstrated efficient mode locking of erbium doped fiber laser using graphene carboxymethylcellulose (CMC) polymer composites. The laser gives out soliton pulse with duration of $\sim 837 \mathrm{fs}$, and $0.19 \mathrm{~nJ}$ pulse energy.

OCIS codes: (140.4050) Mode-locked lasers; (190.4400) Nonlinear Optics, Materials
\end{abstract}

\section{Introduction}

Ultrafast mode locked fiber lasers are useful light sources in optical communication, sensing, metrology, biomedical imaging etc. Due to their simplicity, versatility, efficient heat dissipation, they are regarded as the next generation light sources compared to their solid state counterparts. The development of ultrafast mode locked fiber laser relies on the saturable absorber which induces the mode locking mechanism.

Currently, the dominant technologies for saturable absorbers are based on semiconductor saturable absorber mirrors (SESAM) [1], nonlinear polarization rotation (NPR) [2], and recently carbon nanotubes (CNT) [3] based saturable absorbers.

The recent intensive study on graphene shows that such magic material exhibits optical saturable absorption which has been demonstrated for mode locked lasers [4]. As a zero bandgap material, graphene exhibits wavelength independent saturable absorption which is ideal for laser mode locking. So far, various types of graphene saturable absorber have been demonstrated including substrate transfer deposition [5], optical deposition [6], and in polymer matrix [7]. Among these, the graphene polymer composites are very attractive since their simplicity and easy handling. Very recently, graphene CMC composite has shown efficient saturable absorption which is promising for laser mode locking [8]. In this report, for the first time, we present graphene produced through thermally expanded graphite in CMC polymer composite as mode locker in an erbium doped fiber laser.

\section{Graphene polymer composite preparation}

Graphene has been synthesized by exfoliation of a fluorinated graphite intercalation compound using of thermal shock resulted in expanded graphite [8]. The elemental composition of the expanded graphite according to the $\mathrm{X}-$ ray Photoelectron Spectroscopy was as follows: C, 96.4; F, 2.4; Cl, 0.1; and O, 1.1 at. \%. Thermally expanded graphite is dispersed in $1 \mathrm{wt}$. \% CMC an aqueous solution by ultra-sonication $(35 \mathrm{kHz}, 500 \mathrm{~W}, 30 \mathrm{~min})$ followed by ultracentrifugation $(10,000 \mathrm{~g}, 30 \mathrm{~min})$ to give a stable suspension of graphene flakes (mixture of graphene with different numbers of layers).

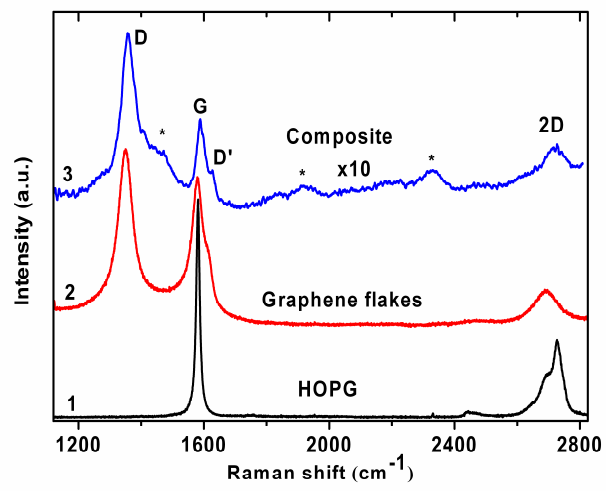

Fig. 1: Raman spectra of (3) composite graphene-CMC, (2) graphene flakes, and (1) HOPG crystals (laser excitation $514 \mathrm{~nm}$ ). *Bands for CMC polymer. 
Films of optical quality were obtained by pouring the graphene suspension on a flat substrate followed by slow evaporation of solvent in a thermostat at $40^{\circ} \mathrm{C}$ for 24 hours. The graphene-CMC polymer composite film has a thickness of $15 \mu \mathrm{m}$ and containing 0.27 wt. \%. graphene in the polymer matrix. Figure 1 plots a Raman spectrum of HOPG (1), graphene flakes on glass (2) and composite graphene-CMC (3). The 2D band on the Raman spectrum of the graphene flakes has a single Lorentzian lineshape with a maximum intensity at $2691 \mathrm{~cm}^{-1}$ and a FWHM of 92

$\mathrm{cm}^{-1}$. For composite graphene-CMC and HOPG, these values for a 2D band are 2709 and 84, and $2723 \mathrm{and}_{32} \mathrm{~cm}^{-1}$, respectively. An estimate of the number of layers in the graphene flakes using the Raman spectrum in Fig.1 (2) gives average values more than four layers.

\section{Fiber laser construction}

Fig. 2 depicts the schematic setup of the graphene CMC polymer mode locked erbium doped fiber laser. The laser consists of $\sim 0.8 \mathrm{~m}$ highly doped erbium fiber as the active medium. A fiber pigtailed optical isolator (OSI) is employed to ensure single direction oscillation of the laser. The laser is pumped by a grating stabilized $975 \mathrm{~nm}$ laser diode through a 980/1550nm wavelength division multiplexing (WDM). A commercial laser diode controller is used as laser diode driver and temperature stabilizer. A standard fused fiber 50:50 coupler is used to extract 50\% optical power.

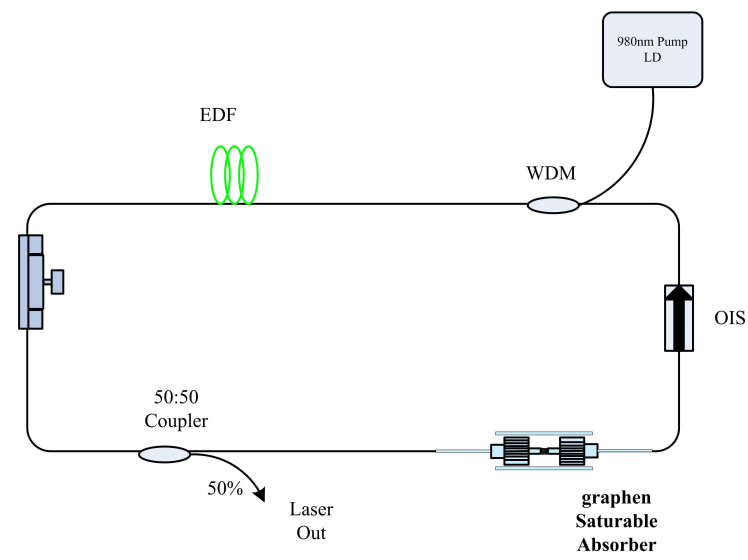

Fig. 2: schematic description of the graphene CMC mode locked erbium doped fiber laser configuration.

An in-line fiber polarization controller is incorporated into the laser cavity to optimize the total birefringence. The graphene CMC polymer composite is sandwiched between two FC/PC connectors by use of the index matching gel in order to minimize the transmission loss. This fiber based mode locker is then spliced into the cavity forming an all fiber mode locked erbium doped fiber laser configuration. The total cavity length is $\sim 7.86 \mathrm{~m}$, corresponding to a net dispersion of $\sim-0.14 \mathrm{ps}^{2}$ indicating the laser will operate in anomalous dispersion regime from which soliton pulse shaping is expected. The output pulse of the fiber laser is fed into an optical spectrum analyzer, a digital oscilloscope and a commercial autocorrelator for characterization.

\section{Fiber laser results}

Fig. 3 shows a typical output optical spectrum of the graphene mode locked fiber laser centered at $1560 \mathrm{~nm}$. The full width half maximum (FWHW) spectral bandwidth is measured to be $\sim 3.52 \mathrm{~nm}$. Clear Kelly side bands indicate soliton pulse shaping which corresponds to our expectation. The laser pulse is then directly fed into the autocorrelator without any amplification. The measured pulse width through the autocorrelator is $\sim 1289 \mathrm{fs}$ corresponding to $\sim 837 \mathrm{fs}$ pulse duration which is shown in Fig.4. A typical pulse train registered by the oscilloscope is shown in Fig. 5 with $\sim 35$ ns pulse interval thus giving a repetition rate of $\sim 28.5 \mathrm{MHz}$. The measured output power is $\sim 5.5 \mathrm{~mW}$ at $\sim 100 \mathrm{~mW}$ pump rendering pulse energy of $\sim 0.19 \mathrm{~nJ}$. The laser has been operated under the laboratory condition for a couple of hours, no visible damage or degradation of laser performance has been observed. 

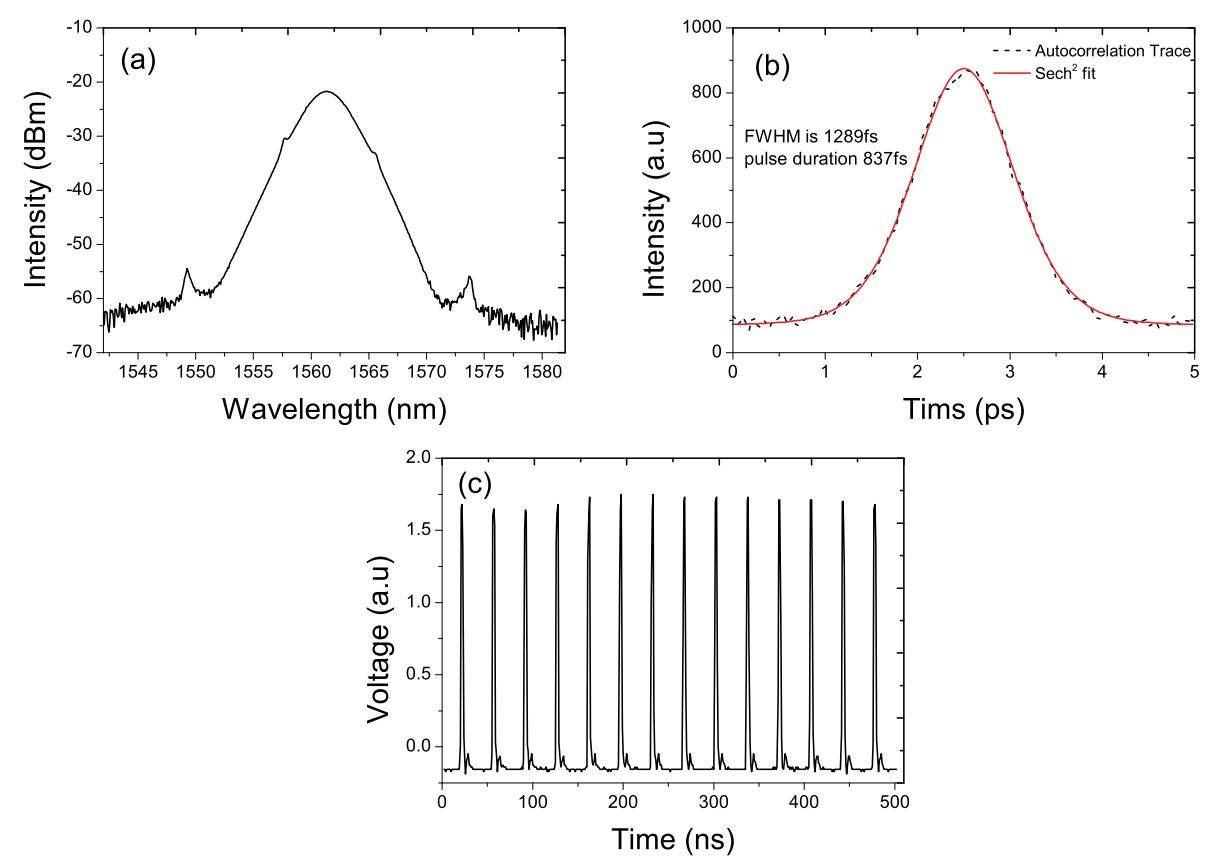

Fig. 3: (a)a typical output optical spectrum with clear Kelly side bands indicating soliton pulse shaping;(b) measured autocorrelation trace of the mode locked laser pulses showing a pulse duration of $837 \mathrm{fs}$; (c) measured pulse trains from the mode locked fiber laser with an repetition rate of $\sim 28.5 \mathrm{MHz}$.

\section{Conclusions}

In conclusion, to the best of our knowledge, for the first time, we present a graphene CMC polymer composite as an efficient mode locker for ultrafast erbium doped fiber laser. The laser has soliton output pulse shape with long term stability under the laboratory conditions. We would also expect the graphene CMC polymer composite could be efficient mode locker for stretch pulse, dissipative soliton and similariton generation.

\section{Acknowledgement}

We acknowledge the partial financial support from the Marie-Curie International Exchange Scheme "TeLaSens", Research Executive Agency Grant \# 269271, Programme: FP7-PEOPLE-2010-IRSES and The Royal Society (Research Grant RG110136).

\section{References}

[1] U.Keller, "Recent developments in compact ultrafast lasers,"Nature.424,831(2003)

[2] H.A.Haus, “Mode locking of lasers,”J.Sele.Top.Quan Elec.6,1173 (2000)

[3] T.Hasan et al, "Nanotube-polymer composites for ultrafast photonics,"Adv.Mater.21,3874(2009)

[4]H.Zhang et al, "Large energy soliton erbium-doped fiber laser with a grapheme-polymer composite mode locker,” Appl.Phys.Lett.95,141103 (2009)

[5] Y.C.Meng et al, "Passively harmonically mode-locked fiber laser with low pumping power based on a grapheme saturable absorber,"Laser Phys.Lett. 9, 537 (2012)

[6] A.Martinez et al, "Optical deposition of grapheme and carbon nanotubes in a fiber ferrule for passive mode-locked lasing," Opt.Express.18,23054 (2010)

[7] D.Popa et al, “sub 200fs pulse generation from a grapheme mode-locked fiber laser,” Appl.Phys.Lett.97,203106 (2010)

[8] D.V.Khudyakov et al, "Saturable absorption of film composites with single-walled carbon nanotubes and graphene,”Applied Optics, 52, 150(2013) 